\title{
Unusually High Rate of Early Failure of Tibial Component in ATTUNE Total Knee Arthroplasty System at Implant-Cement Interface
} \author{
Joshua E. Drumm, DO ${ }^{5}$ \\ 1 Bonutti Clinic, Effingham, Illinois \\ 2 Rubin Institute for Advanced Orthopedics, Center for Joint \\ Preservation and Replacement, Sinai Hospital of Baltimore, \\ Baltimore, Maryland \\ ${ }^{3}$ Department of Orthopaedic Surgery, Mount Sinai West Hospital, \\ New York, New York \\ ${ }^{4}$ Steindler Orthopedic Clinic, lowa City, lowa \\ ${ }^{5}$ Department of Orthopaedic Surgery, Providence Holy Family, \\ Spokane, Washington
}

Peter M. Bonutti, MD ${ }^{1}$ Anton Khlopas, MD² Morad Chughtai, MD² Connor Cole, PA-C ${ }^{2}$ Chukwuweike U. Gwam, MD ${ }^{2}$ Steven F. Harwin, MD ${ }^{3}$ Brent Whited, MD ${ }^{4}$ Didi E. Omiyi, MD ${ }^{5}$

J Knee Surg 2017;30:435-439.

\author{
Address for correspondence Peter M. Bonutti, MD, The Bonutti \\ Clinic, 1303 W. Evergreen Avenue, Effingham, IL 62401 \\ (e-mail: p@bonutti.net).
}

\begin{abstract}
Keywords

- total knee arthroplasty

- aseptic failure

- MAUDE

- Implant-cement interface

A novel design total knee arthroplasty (TKA) system has been introduced to improve patient outcomes and increase longevity. However, we have encountered a high rate of debonding of tibial implant-cement interface. In addition, multiple reports have been filed in Manufacturer and User Facility Device Experience database (MAUDE) with the same mechanism of failure. Therefore, we evaluated: clinical, radiographic, and intraoperative findings of patients who received this system and required a revision surgery, and findings from MAUDE database compiled to this date. We reviewed three hospital databases for patients who had revision TKA for tibial loosening at the implant-cement interface. This yielded 15 cases with a mean age of 61 years (range, 47-84). All patients received a novel knee system at another institution. Radiographic analysis was performed by treating orthopaedist. The MAUDE database was reviewed for reports of aseptic failure. Patients presented with pain on weight bearing, effusion, and decreased range of motion (ROM) within 2 years after surgery. Radiographic evaluation demonstrated loosening of the tibial components in 2 of 15 knees. This included cruciate retaining, posterior stabilized, fixed bearing, and rotating platform bearing designs. Intraoperative findings demonstrated gross loosening of the tibial component at the implant-cement interface. Femoral and patellar components were well fixed. There were 21 reports of tibial loosening at the implant-cement interface in MAUDE database in the past 2 months alone. Numerous other tibial failures were reported; however, the mechanisms of failures were not specified. Tibial component loosening is a rare complication of cemented TKA at short-term follow-up. Several possible reasons include increased constraint, reduced cement pockets, and reduced keel rotational stabilizers. The tibial component, which has greater torsional loads, has lower surface roughness than femoral component. We believe that this complication is
\end{abstract}

received

April 5, 2017

accepted

May 4, 2017

published online

June 7, 2017
Copyright $\odot 2017$ by Thieme Medical Publishers, Inc., 333 Seventh Avenue, New York, NY 10001, USA. Tel: +1(212) 584-4662.
DOI https://doi.org/

10.1055/s-0037-1603756. ISSN 1538-8506. 
underreported due to failure of radiographs to assess loosening. In addition, MAUDE database reporting is not consistent and competing companies cannot provide data on the revised components. In patients who have negative workup for a painful joint, one must consider the diagnosis of debonding.

Total knee arthroplasty (TKA) is believed to be one of the most successful surgical procedures with survivorship of more than $90 \%$ at 12 years after surgery. ${ }^{1,2}$ Septic failures usually occur within the first few years, while aseptic failures are not common during first 2 years, especially in cemented TKAs which are known for their early stability. ${ }^{3-7}$ In an attempt to improve functional outcomes, stability, patient satisfaction, and operating room efficiency, a new TKA system has been recently launched. Extensive research and development efforts have been allocated with multiple resultant patents filled. This new TKA system features a gradually reducing femoral radius, an innovative s-curve design of the posteriorly stabilized cam, tibial base which can be downsized or upsized two sizes versus the insert, novel patella tracking, lighter innovative instruments, and a new polyethylene formulation. These implant properties were purported to potentially produce better stability of the knee in deep flexion, reduce the joint forces, produce better patella tracking, better operative flexibility and efficiency, and implant longevity.

Despite these claims, we have noted a large number of revision cases in a short period of time in patients who received this novel TKA system. These patients mostly presented with the anterior knee pain localized to the tibial plateau. Radiographic evaluation demonstrated early loosening in some of the patients, while no signs of loosening in others. Interestingly, intraoperative findings demonstrated freely mobile tibial components with loosening occurring at the implant-cement interface, unlike most aseptic failures which occur in cement-bone interface. Upon review of the Manufacturer and User Facility Device Experience (MAUDE) database, we came across multiple reports of tibial component loosening. However, these incidences might be underreported, as company representatives from another companies cannot report to MAUDE database.

Therefore, the purpose of this study was to describe (1) the clinical, radiographic, and intraoperative findings of patients from three centers who received this novel TKA system and required a revision surgery, as well as (2) the summary of some findings from MAUDE database compiled to this date.

\section{Methods}

\section{Patient Selection}

We reviewed three hospital databases for patients who had revision TKA for aseptic failure, specifically, for tibial component loosening at the implant-cement interface. All patients received a novel knee system at another institution. This yielded 13 patients (15 knees) with the mean age of 61 years (range, $47-84$ years) and the mean body mass index of $35 \mathrm{~kg} / \mathrm{m}^{2}$ (range, $21-54 \mathrm{~kg} / \mathrm{m}^{2}$ ). The mean time to revision was 19 months (range, 1-34 months). All procedures were performed by four fellowship-trained adult reconstructive surgeons (J.E.D, P.M.B., B.W., and D.E.O.).

\section{Implant Description}

The knee system used in this study was the ATTUNE Knee System (DePuy Synthes). This new TKA system features a gradually reducing femoral radius, an innovative s-curve design of the posteriorly stabilized cam, tibial base which can be downsized or upsized two sizes versus the insert, novel patella tracking, lighter innovative instruments, and a new polyethylene formulation. These implant properties were purported to potentially produce better stability of the knee in deep flexion, reduce the joint forces, produce better patella tracking, better operative flexibility and efficiency, and implant longevity. There were eight knees that had cruciate retaining, and seven knees that had posterior stabilized bearings. Of the seven posterior stabilized knees, five were fixed and two were rotating platform bearing.

\section{Data Collection}

Preoperative findings were obtained from office notes, which included patient demographics, specific type of implant and cement used for primary TKA, time to revision surgery, physical exam findings, and reasons for revision surgery. ROM data were collected from preoperative office visit notes.

Radiographic analysis of anteroposterior (AP) and lateral radiographs obtained during pre- and postoperative visits was performed by treating orthopaedist utilizing the Knee Society Roentgenographic Evaluation and Scoring System 6 for malposition, radiolucencies, and osteolysis.

Intraoperative findings were obtained from surgical notes. Specifically, we assessed the stability of the tibial and femoral components and adherence of cement to the bone and the implant. In addition, intraoperative images were obtained.

MAUDE database was reviewed for cases of aseptic failure of this device. The MAUDE is a database that contains several hundreds of thousands of medical device reports that are associated with deaths, injuries, and malfunctions. It is an online database that is freely accessible and allows for searching of virtually any device. However, this database is a form of passive surveillance, and therefore, has its inherent limitations, including underreporting. ${ }^{8}$ In addition, competing companies cannot provide data on the revised components. Access to this database is obtained from the following link: https:// www.accessdata.fda.gov/scripts/cdrh/cfdocs/cfmaude/search. cfm. Specifically, we assessed cases of loosening at the implant-cement interface. 


\section{Statistical Analysis}

All data were entered into an Excel spreadsheet (Excel, Microsoft Corporation) after removing patient identifiers. All statistical analysis was performed using SPSS version 24 (IBM Corporation). The statistical analysis was primarily descriptive.

\section{Results}

\section{Sample Case 1}

A 64-year-old woman presented to the office with complaints of right knee pain. She had a right TKA performed 5 months previously at another institution. Past medical history included hypertension and hypothyroidism. Clinical examination revealed a well-healed surgical scar, a small effusion, ROM of 0 to 95 degrees, and pain on palpation of tibial plateau. Patient had increasing pain with flexion and ambulation but decreasing pain at rest. Radiographs did not reveal any progressive loosening or osteolysis. Her infection workup was negative (white blood cell [WBC] count, erythrocyte sedimentation rate [ESR], C-reactive protein [CRP], joint fluid cell count with differential, and fluid culture). Patient underwent revision surgery, and intraoperative findings revealed a grossly loose tibial component, and a wellfixed, stable femoral component. The tibial component had piston motion upon flexion and extension of the knee. Upon removal of tibial baseplate, there was no cement adherent to the inferior surface of the prosthesis. All cement was securely attached to the bone. Intraoperative pathology findings and cultures were negative for infection.

\section{Sample Case 2}

A 55-year-old woman presented to our clinic complaining of right knee pain for the past 2 months. She underwent a right TKA 6 months prior to the presentation at another institution. Two months ago, she developed increasing pain and gradual loss of ROM. On physical examination, she had a well-healed surgical scar, a small effusion, ROM of 0 to 90 degrees, and pain on palpation of tibial plateau. She had no signs of tibial component loosening on radiographic evaluation. Her infection workup was negative. Patient underwent revision surgery, and intraoperative findings revealed a grossly loose tibial component and a stable femoral component. Upon removal of tibial baseplate, there was no cement adherent to the inferior surface. All cement was securely attached to the bone. Intraoperative pathology findings and cultures were negative for infection.

\section{Clinical Findings}

All patients were initially asymptomatic, but subsequently, developed increasing pain with weight bearing, small effusions, and gradual loss of active ROM between 1 month and 2 years after index surgery. All patients presented to the office with complaints of pain on the initiation of weight bearing. On physical examination, there was a tenderness to palpation over the tibial plateau on both the medial and the lateral sides. The mean ROM was 101 degrees (range, 40-125 degrees). Most patients had pain with ROM past 90 degrees. All patients were worked up for possible infection with preoperative joint aspirations, complete blood count, CRP, and ESR. A total of eight knees were tested with Synovasure and were negative. All revision surgeries were performed for the aseptic loosening of tibial component. The intraoperative pathology specimens were culture negative and showed less than five WBCs per high powered field.

\section{Radiographic Findings}

Radiographic evaluation of the 15 knees that received this implant demonstrated only $2(13 \%)$ to have evidence of radiolucencies. In the first case, on the AP radiograph, there was evidence of radiolucency in zones 1 and 2 . On the lateral radiograph, there was evidence of radiolucency in zone $3 \mathrm{~A}$. For the second case, there was evidence of radiolucencies in zones 1, 2, and 3L on AP radiograph, and zones 1, 2, and 3A on lateral view (-Fig. 1).

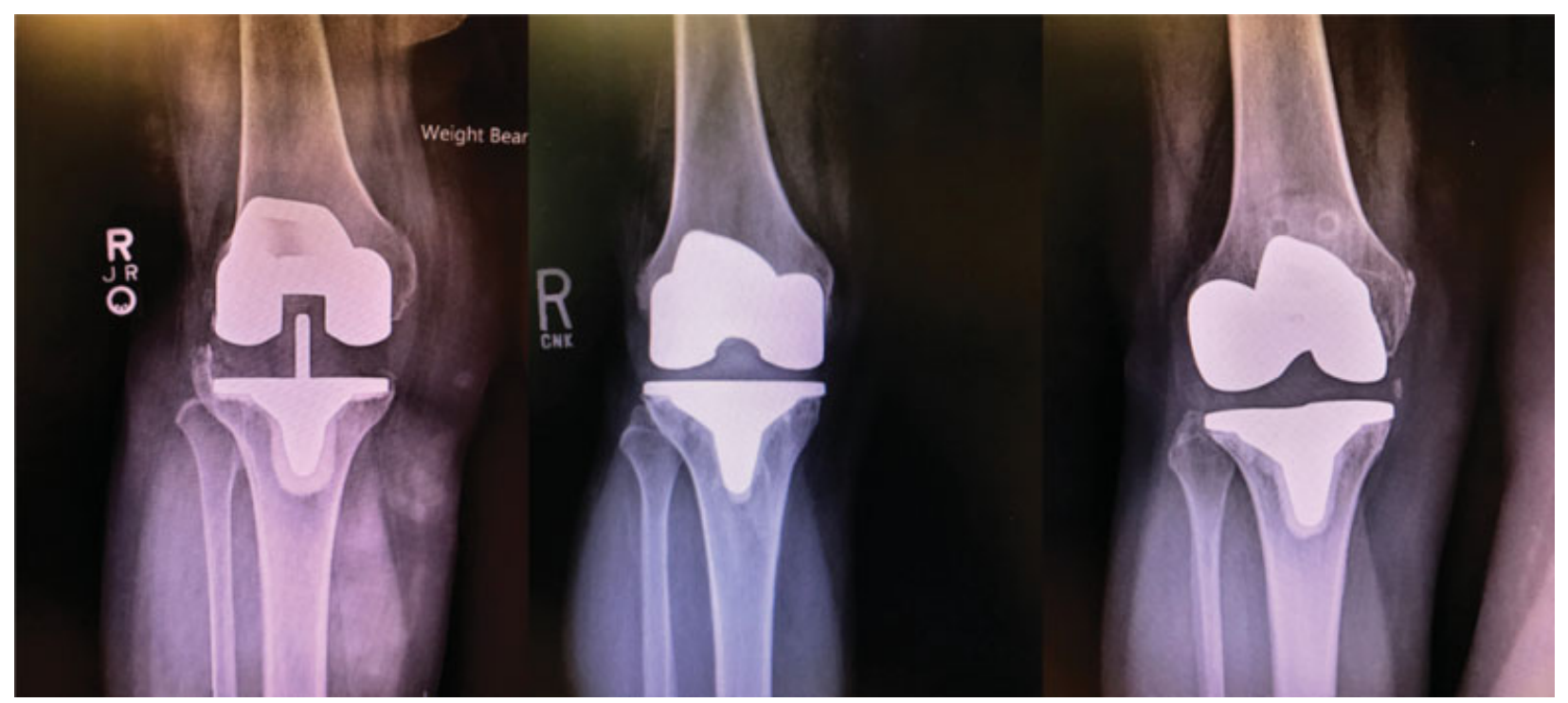

Fig. 1 Anteroposterior radiographs prior to revision surgery. 


\section{Intraoperative Findings}

In all revision cases described in this study, there was an apparent loosening of the tibial component with gross implant motion between the tibial tray and tibial bone cement. In one patient, the tibial tray would piston $4 \mathrm{~mm}$ as the knee was brought from flexion to extension, and would lift off from the tibial bone cement when the leg was manually distracted intraoperatively. All patients underwent revision of both tibial and femoral components. In all tibial cases, the tibial component had debonded and was easily separated from the cement mantle. Upon removal of the tibial baseplate, the inferior surface of the tibial baseplate was clear of cement ( - Fig. 2), while all the cement was strongly adherent to the tibial bone (-Fig. $\mathbf{3}$ ). The femoral components were securely attached to the femoral bone with no apparent loosening. Upon explantation of the femoral components, the cement was strongly adherent to the implant surface in all cases ( - Fig. 4).

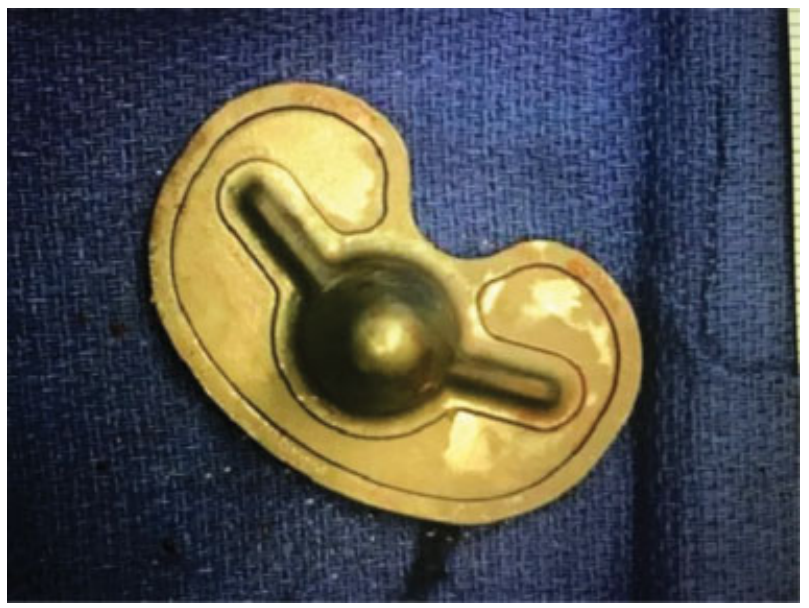

Fig. 2 Inferior surface of the tibial component immediately after explantation.

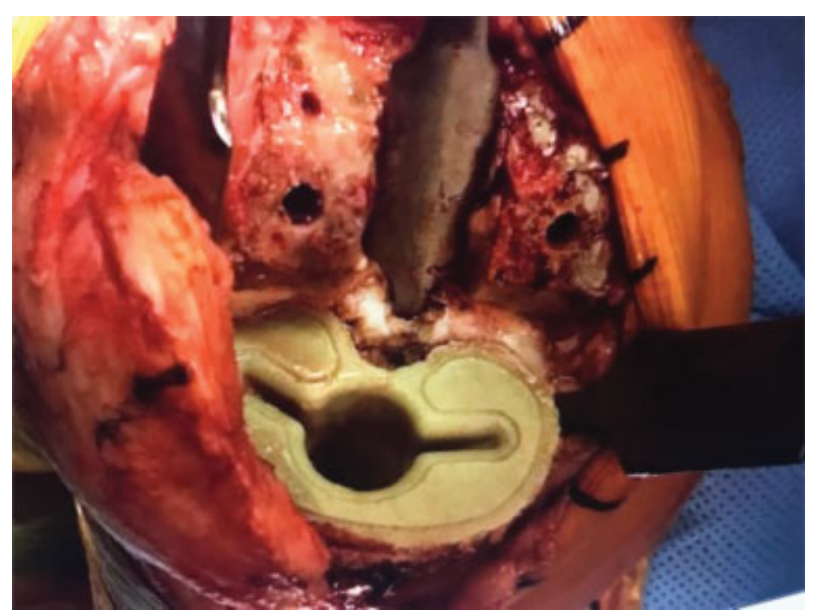

Fig. 3 Cement adherent to the tibial bone after explantation of tibial component.

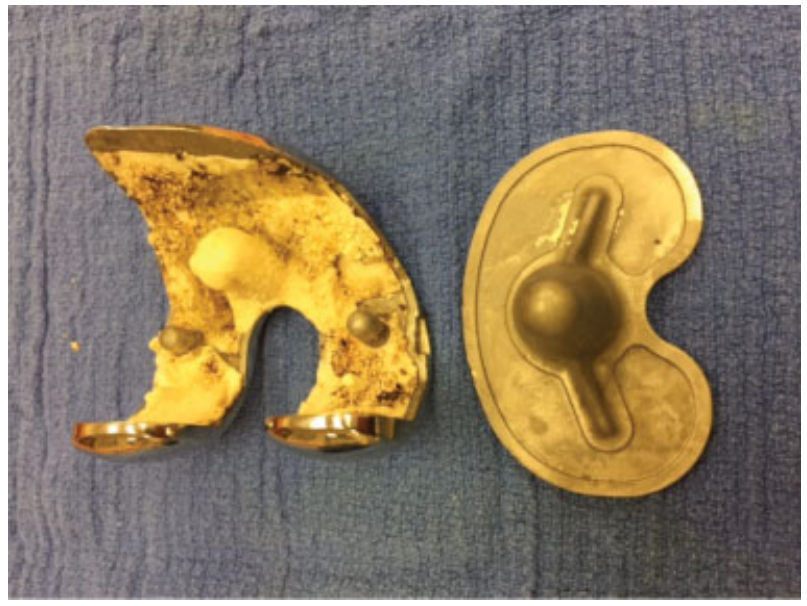

Fig. 4 Cement adherent to femoral component but not tibial component.

\section{Manufacturer and User Facility Device Experience Database}

To assess the global reported occurrence of this type of failure, we entered brand name of the device into the search engine of the MAUDE database. A search of the past 2 months (January and February 2017) yielded 204 reports on this device. Of those, there were 21 reports of aseptic failure due to debonding of implant-cement interface of the tibial component.

\section{Discussion}

A new primary knee system with innovative design has been recently introduced in an attempt to improve the patient outcomes and to increase the implant longevity. Several studies have described good short-term outcomes of this prosthesis in a setting of primary TKA. ${ }^{7}$ In our experience of three community hospital centers, we have encountered unusually high numbers of the aseptic early failures. In addition, many reports have been filed in MAUDE database concerning tibial loosening at the implant-cement interface. ${ }^{8}$ Numerous other tibial failures were reported, however, the mechanisms of failures were not specified. Furthermore, there appears to be a similarity in the mechanism of failures. There was a failure of cement-to-implant interface in a tibial component of this implant in all cases reported in this study. Femoral component, on the contrary, retained its stability in all cases. Interestingly, most of the patients had negative findings on radiographic evaluation, which can be attributed to the unusual mechanism of failure through debonding of the implant-cement interface.

Several possible reasons for increased failure of this implant include increased constraint of tibial polyethylene, reduced cement pockets in tibia, reduced rotational stabilizers on keel (i.e., all smooth surfaces on tibial fixation surface), and roughness factor on fixed bearing is only 60 versus 220 on the previous generation system (Sigma, DePuy Synthes). The increased constraint of the tibial poly may contribute to higher loads at the implant-cement and 
cement-bone interfaces, which may contribute to the loosening. The design of the inferior surface of the tibial component appears to have rounded edges and less insets for cement interdigitation relative to the prior designs. In addition, the tibial keel appears to have less rotational phalanges which may lead to less rotational stability and less macrointerdigitation. Furthermore, according to the manufacturing literature, the backside surface of the tibial component has markedly different surface roughness when compared with the femoral component (60 vs. 220 grit blasts). All of these factors may contribute to the propensity of this implant for early loosening through higher loads and less stability at the tibial implant-cement interface.

\section{Conclusion}

A new TKA system with novel design has been recently introduced and showed good early outcomes in a prospective study after 2-year follow-up. However, in our experience of three community hospital centers, we have encountered an unusually high rate of early aseptic failures as a result of failure of implant-cement interface. In addition, multiple reports have been filed in MAUDE database concerning tibial loosening at the implant-cement interface. After carefully examining the implant design, we have proposed several possible reasons for this early aseptic loosening, which include increased constraint, reduced cement pockets, reduced keel rotational stabilizers, and reduced surface roughness of the tibial component when compared with the previous generation implant. Although we report on only 15 cases, we believe that this complication is markedly underreported due to failure of radiographs to accurately assess loosening. In addition, MAUDE database reporting is not consistent by the company representatives and competing companies cannot provide data on the revised components. In patients who present with unexplained pain and undergo a thorough workup for a painful joint, which includes blood work and imaging, one must consider the diagnosis of debonding at implant-cement interface. Larger retrospective studies with longer follow-up are warranted to further investigate the prevalence of aseptic failure in these implants.

\section{References}

1 Graves SE, Davidson D, Ingerson L, et al. The Australian Orthopaedic Association National Joint Replacement Registry. Med J Aust 2004;180(5 Suppl):S31-S34

2 Abdel MP, Morrey ME, Jensen MR, Morrey BF. Increased long-term survival of posterior cruciate-retaining versus posterior cruciatestabilizing total knee replacements. J Bone Joint Surgery Am 2011;93(22):2072-2078

3 Berend ME, Ritter MA, Meding JB, et al. Tibial component failure mechanisms in total knee arthroplasty. Clin Orthop Relat Res 2004;(428):26-34

4 Sharkey PF, Lichstein PM, Shen C, Tokarski AT, Parvizi J. Why are total knee arthroplasties failing today-has anything changed after 10 years? J Arthroplasty 2014;29(09):1774-1778

5 Healy WL, Della Valle CJ, Iorio R, et al. Complications of total knee arthroplasty: standardized list and definitions of the Knee Society. Clin Orthop Relat Res 2013;471(01):215-220

6 Meneghini RM, Mont MA, Backstein DB, Bourne RB, Dennis DA, Scuderi GR. Development of a modern Knee Society Radiographic Evaluation System and methodology for total knee arthroplasty. J Arthroplasty 2015;30(12):2311-2314

7 Ranawat CS, White PB, West S, Ranawat AS. Clinical and radiographic results of ATTUNE and PFC Sigma knee designs at 2-year follow-up: a prospective matched-pair analysis. J Arthroplasty 2017;32(02):431-436

8 MAUDE - Manufacturer and User Facility Device Experience. Available at: https://www.accessdata.fda.gov/scripts/cdrh/cfdocs/cfmaude/ search.cfm. Accessed April 4, 2017 